Desarrollo de los procesos investigativos en el Departamento de Biología de la Universidad Pedagógica Nacional. Aportes a la producción de conocimiento, la formación docente y la

\title{
proyección social
}

\author{
Por: Alice Marcela Gutiérrez P., Carlos Arturo Sierra D., Edgar Orlay Valbuena U. \\ galice_marcela@hotmail.com \\ carlattasierra@hotmial.com \\ edgarorlay@hotmail.com
}

Presentamos las tendencias de la investigación en el Departamento de Biología (DBI) de la Universidad Pedagógica Nacional (UPN), en lo que atañe al objeto de estudio y las implicaciones tanto en la producción de conocimiento, como en los aportes a la formación docente y la proyección social. Hacemos énfasis en el periodo de los últimos diez años y esbozamos algunas características de las Líneas de Investigación consolidadas en este periodo de tiempo. Todo ello, sin descuidar los antecedentes que consideramos constituyeron el caldo de cultivo para alcanzar los logros actuales.

La dinámica de investigación en el DBI ha cambiando, así, en un comienzo estaba soportada principalmente en los trabajos de grado de los futuros profesores, y en la actualidad se desarrolla al interior de grupos y líneas de investigación lideradas por los formadores de formadores. En cuanto a los objetos de investigación, éstos son diversos y han tenido un giro, pasando de tendencias en la disciplina biológica a problemas relativos a la educación, la pedagogía y la didáctica de la Biología.

Las investigaciones del DBI han aportado elementos fundamentales en la formación de docentes, al constituir la base para la formulación de Proyectos Curriculares de pregrado (Licenciatura en Biología) y de postgrado (Especialización en Enseñanza de la Biología). A su vez, dichos Proyectos Curriculares, especialmente el de pregrado, se desarrolla desde la perspectiva de la investigación formativa y constituye un sustrato para el fortalecimiento y/o la constitución de Líneas de Investigación.

Pese a que los diferentes grupos de investigación del DBI han desarrollado trabajo con comunidades educativas y con profesores de ciencias y de Biología, en el contexto de cursos de actualización, programas de formación permanente de docentes y seminarios, en los ámbitos local, nacional e internacional, consideramos que podríamos aprovechar aún más los logros alcanzados desde de las diferentes investigaciones.

Palabras Clave: investigación, formación docente, Proyecto Curricular de Licenciatura en Biología, Enseñanza de las Ciencias, Pedagogía, Didáctica. 


\section{Agentes dinamizadores de la investigación en el DBI -Un vistazo a las décadas de los 80 y 90}

La historia de las ciencias nos ha enseñado que el estado de ella, en un momento dado es el resultado de una convergencia de múltiples caminos, enfoques y condiciones. Esto también es válido para entender la actualidad del estado de investigación en el DBI. No hay una ruta continua y recta, sino ires y venires, desviaciones y reencuentros, cambios en el nivel de análisis, reformulaciones y readecuaciones metodológicas.

Lo anterior nos ha llevado a reconocer diferentes dinámicas que se han desarrollado en relación a la investigación y la formación de Licenciados en Biología. Sin embargo, no queremos iniciar este recorrido sin hacer antes referencia a algunos aspectos que antecedieron y facilitaron espacios para la reflexión académica en torno a la misión y visión del DBI, durante los últimos diez años.

En la década del 70, la formación de Licenciados en Biología, estuvo centrada en la 'Preparación metodológica para organizar y administrar contenidos', un acercamiento a la 'Ciencias de la educación' (psicología, sociología y filosofía de la educación) y un marcado acento en los saberes de la Biología. La investigación era algo realizado por unos pocos y lejos del entorno inmediato.

Con la reforma del plan de estudios en la década del 80, se incluyó como uno de los objetivos de formación "Desarrollar aptitudes para la investigación", y en los fines que se "...puedan realizar investigación, docencia y aplicar sus conocimientos científicos en la solución de problemas nacionales" (DBI, 1984: 5). El Plan estaba basado en la estructura conceptual de la Biología, áreas de integración y niveles de complejidad y organización, lo que implicaba un trabajo de análisis de la historia, la epistemología, la pedagogía y didáctica y aplicabilidad de los conceptos que permitían dar cuenta de los hechos u objetos de conocimiento abordados. Este enfoque permitió vislumbrar una manera distinta de pensar las relaciones que emergen en el quehacer del docente, posibilitó entre otras cosas el desarrollo de trabajos, que aunque incipientes, mostraban una dimensión distinta al mero dictar clases y abría la puerta a la investigación educativa como parte integral de la actividad del docente. Se introdujo el trabajo de grado como requisito parcial para optar al título de licenciados.

La intencionalidad declarada en el documento marco del Plan de Estudios de 1984, era básicamente formar a los futuros maestros en la investigación pedagógica y didáctica. Sin embargo, los profesores formadores, en su mayoría, no estábamos preparados para esa tarea y nos refugiamos en la disciplina biológica para hacer los pinitos en investigación, orientando una enorme cantidad de trabajos de grado, que cubrían una amplia gama de temáticas, enfoques y problemáticas. Esto nos permitió instaurar una tradición que, con 
diferentes estilos de trabajo, nos condujo a formar grupos alrededor de temáticas u orientaciones metodológicas y epistemológicas.

Las continuas discusiones académicas, en el marco del Movimiento Pedagógico, nos movilizó conceptualmente y redireccionamos los esfuerzos hacia la búsqueda de una identidad como Departamento, desde lo disciplinar y lo pedagógico en un sistema de ideas que se fue configurando como saber escolar. La particularidad de nuestra profesión empezó a ser entendida como un complejo multidimensional en el que el quehacer cotidiano involucraba los saberes de la disciplina a enseñar, la reflexión sobre las relaciones emergentes en la actividad docente, el análisis de la lógica, la historia y la filosofía de las ciencias como marco para la construcción de los conceptos y el desarrollo de las potencialidades de los estudiantes. Hubo un desplazamiento del centro de atención, ahora lo importante ya no era sólo el estudiante o el maestro sino la construcción del conocimiento por los actores involucrados en el proceso y por ello, fue importante estudiar las condiciones que afectaban esa construcción. Se hizo necesario, entonces, indagar sobre las preconcepciones, nociones o preconceptos que tenían, tanto alumnos como maestros, se buscó caracterizar las estrategias didácticas más efectivas, es decir, hubo un cambio de mirada.

Poco a poco, la investigación se fue constituyendo en un ejercicio de la actividad docente en el DBI. A mediados de la década del 90, era el centro, el eje de la formación de los futuros maestros y el DBI, tenía ya varios grupos de investigaciones consolidados, con líneas y proyectos en desarrollo. Sin embargo, Puerto y Rodríguez (1995), hacen la caracterización de de las tendencias de 110 proyectos de grado realizados entre 1989-1994 encontrando que: sólo 13 de los trabajos analizados se podían ubicar fácilmente en una de las cuatro líneas, existentes en ese entonces (Enseñanza de la Biología en Colombia, Faunística y biodiversidad, Estudios en Calidad de Vida, Biodiversidad y conservación de los sistemas acuáticos del Santuario de Flora y Fauna de Iguaque). Las temáticas abordadas, según las autoras eran: didáctica (75), biología (23), comunidad (7), historia (3), filosofía (2).

Es interesante anotar que el énfasis en lo didáctico se mantuvo durante ese periodo (representaba alrededor del 20\% de los trabajos, excepto en 1992, cuando bajó a un 8\%, mientras que el interés por lo disciplinar fue creciendo paulatinamente desde un $4.3 \%$ en el 89 hasta alcanzar el 43\% en 94. Lo anterior, nos llevó a tener que visualizar y proyectar el trabajo formativo y de investigación, pues la pregunta que planteábamos era: ¿Estamos descuidando la formación pedagógica y sobrevalorando lo disciplinar de la Biología?

\section{Desarrollo de la investigación en el BDI -La última década 1997-2007-}

Teniendo en cuenta el contexto anterior, el DBI tenía un reto académico, pues la idea era formar Licenciados en Biología que reconocieran su profesión más allá de la disciplina, 
dándole un espacio de análisis y construcción a la formación pedagógica y didáctica desde la investigación educativa.

“El comité de práctica, entre los años 95 y 98, realizó debates en torno a la formación de los Licenciados en Biología y el papel que juegan allí las prácticas pedagógicas. Este proceso que se inició con la investigación "Análisis de las Características del Maestro en Formación de la Licenciatura en Biología" (Castaño, et al, 1998), en la que se consideraban como puntos críticos a considerar en la formación del docente en Biología, el poco interés por la pedagogía y la falta de identificación con la profesión docente. Consideraba que estos aspectos se hacen más críticos cuando se desconocen sistemáticamente las características y creencias de los estudiantes en relación con aspectos afectivos y culturales, tales como el sentido que le dan a sus vidas, su visión de país, la imagen que tienen de la profesión docente y de la educación; las relaciones que encuentran entre la disciplina biológica y la formación pedagógica, entre otros aspectos" ${ }^{\prime *}$.

Es así como el nuevo proyecto curricular se viabiliza gracias a dos situaciones, una relacionada con el acontecer del Departamento y su experiencia en el desarrollo de la propuesta de formación de los Licenciados en Biología. La otra con el planteamiento que se hace desde el Proyecto Político Pedagógica de la UPN en el año de 1997, el cual aporta el cuerpo fundamental y marco conceptual para la reestructuración de las propuestas formativas al interior de cada Departamento. Estas situaciones, llevan al DBI a buscar espacios para el encuentro académico que le permita replantearse la idea del Licenciado en Biología que quiere formar, llegando así al año 2000.

Estas intenciones se objetivaron en la estructuración del, actual, Proyecto Curricular Licenciatura en Biología (PCLB), en el que la dimensión investigativa es el eje articulador: cada Eje curricular o semestre se organiza con Núcleos integradores de Problemas (NIP), para ser resueltos por los estudiantes con el concurso de los docentes de todas las asignaturas o componentes que hacen parte del semestre. Estamos convencidos de que la educación formal debe pretender una formación integral, abandonando la atomización de saberes y adoptando un enfoque sistémico, y por lo tanto interdisciplinario, que posibilite al estudiante la construcción de una visión integral y dinámica de la naturaleza y la formación y desarrollo de un verdadero "espíritu científico"; lo que nos puso en otro nivel de análisis que implicaba el uso de la investigación pedagógica, didáctica y en la biología como elementos formativos del maestro formador, desde los cuales se podría organizar el currículo con una perspectiva que respondiera a las demandas sociales, del Ministerio y de la UPN, pero también a la lógica y estructura de la disciplina.

Esta situación se toma como un indicador de la necesidad de identificar otros enfoques y alternativas en la enseñanza de las diferentes disciplinas, de tal forma que los estudiantes

\footnotetext{
* Declaración de la profesora Norma Constanza Castaño, Coordinadora del comité de práctica del Programa de Licenciatura en Biología, de ese entonces.
} 
logren un mayor nivel de aprendizaje significativo y comprensivo y así se puedan maximizar las posibilidades de éxito en un mundo cambiante y veloz.

De esta forma, en el desarrollo del (PCLB), se suscitan nuevas preguntas que encontrarán nuevas posibilidades de respuestas en maestros preparados para el abordaje, la nueva perspectivas lleva a los grupos a viabilizar las problemáticas a través de proyectos de investigación, que son posibles gracias a los Proyectos de Facultad, Centro de Investigaciones de la Universidad Pedagógica (CIUP), Instituto para la Investigación y el Desarrollo Pedagógico (IDEP), Conciencias y el tiempo que se asigna en la carga horaria de los profesores ocasionales y catedráticos.

Las condiciones están dadas y las posibilidades de hacer investigación nos llevan a desarrollar investigaciones que dan posibilidades de consolidar grupos y/o líneas de investigación. A continuación, presentamos una breve descripción de las, principales finalidades de las 11 líneas de investigación que actualmente hacen parte del DBI, de acuerdo con la información dada por cada uno de los grupos:

Biotecnología y Educación: proyectar socialmente el análisis crítico y la reflexión de los adelantos de la biotecnología, diseñando estrategias que faciliten la enseñanza / aprendizaje de las ciencias, promoviendo la alfabetización científica que facilite la comprensión de las relaciones C/T/S.

Desarrollo Sostenible en el Sector Rural: sensibilizar a las Comunidades Educativas del sector rural, hacia la conservación y protección del Sistema Ambiental Local bajo los principios de la sostenibilidad. Haciendo especial énfasis en el escolar campesino, para que éste tome actitudes favorables, hacia la protección y conservación de la vida.

Estudios Ecológicos para la Conservación de los Sistemas Acuáticos de la Región Andina: sus principales propósitos son: caracterizar físico - químicos y biológico de los sistemas acuáticos de esta región. Realizar estudios orientados a evaluar la calidad biológica de los sistemas acuáticos de esta región. Proponer procesos de cualificación de docentes de básica y media de los planteles educativos de esta región, en aspectos relacionados con el conocimiento de la biodiversidad, conservación y manejo sostenible de los recursos hídricos.

La Educación Ambiental en el Contexto Educativo Colombiano: construir conocimiento sobre Educación Ambiental en aspectos conceptuales, pedagógicos, metodológicos y didácticos a partir de los desarrollos teóricos y la identificación de los enfoques y tendencias en el campo en los diferentes contextos educativos, particularmente en la escuela.

Estudios en Calidad de vida y Probleduciencias: el principal objetivo del trabajo tanto en calidad de vida como en probleduciencias es la producción de conocimiento en cada campo. En calidad de vida se logra, mediante el diseño, ejecución, evaluación y propuesta de estrategias de contribución al mejoramiento de la calidad de vida y el desarrollo 
humano a través de la educación así como de contribución a la generación de políticas pertinentes a su objeto. Mientras que en probleduciencias, la producción se centra en el campo de la didáctica, con el fin de contribuir a la consolidación de una postura teórica unificada pero flexible, en el ámbito de la enseñanza de las ciencias, la educación centrada en la resolución de problemas, a partir del modelo "Resolución de Problemas en una Perspectiva de Investigación".

Faunística y Conservación de Artrópodos: generar conocimiento, actividades educativas y aplicaciones pedagógicas en torno a la artropofauna contribuyendo con ello a su conservación y utilización, mejorando así la calidad de vida de los colombianos. Así mismo, adaptación, implementación y validación de propuestas pedagógicas, alrededor de la ecología, conservación y educación ambiental, utilizando preferiblemente insectos.

Conocimiento profesional del profesor de ciencias: reflexionar en torno a la construcción de este tipo de conocimiento, el cual caracteriza al docente de Ciencias Naturales y lo diferencia del quehacer de otros profesionales. En ese sentido, pretende producir conocimiento pedagógico y didáctico relacionado con las fuentes, los componentes y las relaciones que hacen posible la construcción de dicho conocimiento, el cual posee un estatus epistemológico diferenciado, que le permite al docente integrar y transformar los saberes que confluyen en la enseñanza de las Ciencias.

Ciencias, Acciones y Creencias: trabajar acerca de las creencias de los profesores de ciencias sobre la naturaleza de la ciencia, la enseñanza y el aprendizaje; y la identificación de algunas implicaciones de estas creencias en la enseñanza y en el aprendizaje. También, trabaja en la experimentación de modelos alternativos para mejorar la enseñanza de las ciencias que produzcan impactos positivos en el aprendizaje de los estudiantes sobre: los conceptos científicos, las prácticas de laboratorio, la resolución de problemas, el mejoramiento de las actitudes hacia el aprendizaje y el análisis crítico de las relaciones ciencia - tecnología y sociedad.

Pensamiento del Profesor: cuestionar, construir y transformar conocimientos educativos a partir de las reflexiones sobre la enseñanza de la Biología y las Ciencias Experimentales. Interpretando y caracterizando varias de las principales acciones y problemáticas que afrontan los profesores en la Educación Básica Secundaria, desde fundamentos pedagógicos y didácticos actuales para contextos de realidades colombianas.

Trayectos y Aconteceres: Estudios del Ser y el Quehacer del Maestro desde la Pedagogía: aportar a la construcción de un saber propio de la pedagogía desde la indagación histórica de las prácticas y los discursos sobre educación, enseñanza, aprendizaje, maestro, estudiante, escuela, saber pedagógico y disciplinas de enseñanza. Además, de establecer las condiciones de posibilidad de algunas prácticas y discursos relacionados con la pedagogía. 
Desarrollo del Pensamiento Biológico y de la Comunicación en el Niño: caracterizar las formas de pensar de los niños acerca de lo vivo y construir un modelo que permita comprender el desarrollo cognitivo y propiciar aprendizajes en esta área de conocimiento. Busca también, comprender las interacciones que se suceden en el aula de clase de ciencias en lo que respecta a los diversos saberes construidos acerca de lo vivo y los diferentes tipos de discurso para la expresión de dichos saberes y las interacciones que enmarcan el trabajo en el aula de clase.

\section{¿Cuál ha sido el objeto de investigación en el Departamento de Biología?}

Las investigaciones realizadas durante los últimos diez años en el DBI, ya sea en el contexto de grupos o líneas de investigación se ocupan de diversos problemas. En la Tabla No. 1 se agrupan los estudios realizados, de acuerdo con los objetos y problemas de investigación; igualmente se presentan los títulos de algunos de los proyectos realizados (financiados por el Centro de Investigaciones de la Universidad Pedagógica Nacional CIUP-, Colciencias y el IDEP. En su mayoría por el CIUP) y las referencias de algunas publicaciones gestadas a partir de los diferentes proyectos. Cabe señalar que debido a la limitación en el espacio disponible, el presente artículo referencia tan sólo una muestra de las producciones de los grupos de investigación del DBI.

A partir de la tabla, podemos reconocer que una cantidad significativa de investigaciones, atañen al campo de investigación en Enseñanza-aprendizaje de las Ciencias y de la Biología. Los estudios al respecto se refieren a cinco dimensiones:

-Los referentes históricos y epistemológicos de la Biología y de la enseñanza de esta ciencia en Colombia.

-La educabilidad y enseñabilidad de áreas referentes a temáticas contemporáneas en la Biología, como son la salud, la conservación de la biodiversidad, la Ecología y Biotecnología.

-La enseñabilidad de temáticas específicas, concretamente de conceptos de Botánica -Modelos y estrategias para la enseñanza de las ciencias y de la Biología, tales como la resolución de problemas y la utilización de las nuevas tecnologías de comunicación.

-Análisis de representaciones y de pensamiento acerca de aspectos biológicos, tal es el caso de las investigaciones sobre el pensamiento de los niños sobre lo vivo, y de las representaciones sociales acerca microorganismos.

La realización de dichos estudios corresponden principalmente a las líneas de investigación: Pensamiento del Profesor, Estudios en Calidad de Vida, Estudios Ecológicos para la Conservación de los Sistemas Acuáticos, Faunística y Conservación de Artrópodos, y Biotecnología y Educación. Es de resaltar que los resultados de estas investigaciones, además de contribuir en la producción de conocimiento pedagógico y didáctico, han aportado importantes elementos para la estructuración de Proyectos Curriculares tanto de pregrado (de Licenciatura en Biología) como de postgrado (Especialización en Enseñanza 
de la Biología), y se han constituido en el pilar para la realización de eventos educativos de orden local, nacional (Encuentros de Enseñanza de la Biología y la Educación Ambiental, Diplomados dirigidos a maestros del Distrito y Secretaría de Salud, así como encuentros de Experiencias en Biotecnología en los niveles educación básica y media) e internacionales (Cursos Internacionales de Educación para la Conservación en el geotrópico UPN-OEA-ICETEX).

Uno de los campos en los que más se ha investigado en el DBI es en el de las creencias, pensamiento y conocimiento profesional del profesor de ciencias y de Biología. Al respecto la producción ha sido nutrida en lo que tiene que ver con la caracterización de las concepciones y del pensamiento de docentes en ejercicio, y derivado de ello las implicaciones en la formación permanente de docente, y la formulación y desarrollo de propuestas para el mejoramiento de las prácticas pedagógicas. En los dos últimos años venimos desarrollando investigaciones con futuros profesores de Biología, dichos estudios están orientados a caracterizar sus concepciones acerca de diferentes componentes del Conocimiento Profesional del profesor de Biología; además estamos identificando los elementos formativos al interior del Proyecto Curricular de Licenciatura que contribuyen a la construcción y reconstrucción de dicho Conocimiento Profesional.

La caracterización de la formación docente en el DBI, se reconoce desde dos puntos de vista: uno relacionado con el aporte que desde la investigación se hace a la cualificación de docentes en ejercicio, encontrado que este campo es propicio y oportuno para la proyección social desde la investigación que hacen algunas líneas, como por ejemplo: serie de conversatorios de Trayectos y Aconteceres, los cuales se han ido desarrollando desde hace varios años, talleres que desde Ciencia, Acciones y Creencias y Pensamiento del Profesor se han llevado a cabo con docentes en formación y del Distrito Capital, entre otros.

El otro, esta relacionado con la flexibilidad que tiene el PCLB, en cuanto a la posibilidad que se tiene para abordar diferentes retos y problemáticas, los cuales se van reconociendo en el desarrollo del mismo. Desde esta perspectiva, se han concebido y desarrollado varias investigaciones que responden a la formación docente, con una mirada de reconocimiento y autoevaluación tanto de la propuesta curricular, como lo son los "proyectos de evaluación del proyecto curricular fases I y II" ; así como de los estudiantes, desde proyectos como: "Las condiciones de autonomía de los estudiantes del Proyecto Curricular Licenciatura en Biología" y "Análisis de las Características del Maestro en Formación de la Licenciatura en Biología", entre otros. Los resultados de estas investigaciones han permitido recocernos y plantear proyecciones al interior del Departamento en aras de mejorar la calidad en la formación de los futuros maestros.

El campo de investigación relacionado con la pedagogía, hace énfasis en la reflexión y el sentido que se le da a esta ciencia como saber propio de los maestros, es otra de las características que desde diferentes líneas e investigaciones se le ha dado a esta área de 
saber. Investigaciones como las realizadas, en su momento desde violencia y escuela, en donde se establecieron los imaginarios de violencia presentes en la relación maestro estudiante, que dieron paso al planteamiento de otros trabajos relacionados con la práctica pedagógica en la generación, trasformación y y/o disminución de la violencia en la escuela. Los aportes desde este campo le han permitido al Departamento rescatar al maestro como profesional, estudiándolo desde su ser y su quehacer en el ámbito pedagógico.

Cabe destacar que los diferentes grupos de investigación, además de haber publicado artículos y libros, hemos socializado los resultados de los estudios en numerosos eventos nacionales e internacionales, entre los que destacamos: Congresos de la Asociación Colombiana de Ciencias Biológicas, Congresos Internacionales de Enseñanza de la Biología organizados por la Asociación de Docentes de Ciencias Biológicas de Argentina, Congresos Internacionales de profesores de Ciencias organizados por la Revista TED, Congresos Internacionales de Enseñanza de las Ciencias organizados por la Revista Enseñanza de las Ciencias, Congresos Nacionales de la Sociedad Colombiana de Entomología -SOCOLEN-, Congresos Internacionales de Microbiología Ambiental y Microbiología Industrial organizados por la Pontificia Universidad Javeriana de Bogotá, ,

Otro aspecto que no queremos dejar pasar desapercibido es el aporte de la investigación del DBI en los procesos de formación inicial de docentes. Así, el enfoque complejo y la estructura del Proyecto Curricular de Licenciatura en Biología (PCLB) obedece a las contribuciones de las Líneas de investigación, lo que conlleva a concebir dicho proyecto como una investigación de autoevaluación formativa. Los énfasis del Ciclo de Profundización del PCLB son formulados y desarrollados fundamentalmente con base en cuatro líneas de investigación. De otra parte, los problemas que abordan los futuros licenciados en Biología su práctica pedagógica integral (en el Ciclo de Fundamentación del PCLB) son orientados por los diferentes grupos de investigación. De igual manera, los proyectos pedagógicos desarrollados por los docentes en formación, en el marco de la práctica docente en el Ciclo de Profundización, son realizados al interior de proyectos de investigación de las once Líneas. Es de resaltar que todos los trabajos de grado son realizados por los estudiantes del PCLB al interior de los grupos de investigación, y generalmente contribuyen al cumplimiento de objetivos de investigaciones puntuales. Una tendencia en los últimos años es que los trabajos de grado correspondan a la continuación de los proyectos pedagógicos desarrollados en la práctica docente.

\section{Conclusiones}

- El reconocimiento del contexto que aconteció a la investigación, que hoy en día se desarrolla en el DBI, permite dar razón de las trasformaciones significativas en cuanto a la investigación, la producción de conocimiento y la formación de Licenciados en Biología.

- La investigación en el DBI, ha permitido construir conocimiento, en las áreas disciplinar y pedagógica y didáctica. 
- A través de la investigación se ha fortalecido la propuesta de formación de Licenciados en Biología tanto en pregrado como en postgrado.

- El reto que enfrentamos hoy en día como comunidad académica, es avanzar en la proyección social, teniendo en cuenta los logros alcanzados en nuestras investigaciones. 
Tabla No. 1 "Agrupación de las investigaciones del Departamento de Biología de acuerdo con los campos y objetos de estudio"

\begin{tabular}{|c|c|c|c|}
\hline $\begin{array}{l}\text { CAMPOS DE } \\
\text { INVESTIGACIÓ } \\
\mathbf{N}\end{array}$ & $\begin{array}{l}\text { OBJETOS DE } \\
\text { INVESTIGACIÓN }\end{array}$ & $\begin{array}{c}\text { ALGUNAS INVESTIGACIONES } \\
\text { REALIZADAS }\end{array}$ & $\begin{array}{c}\text { ALGUNAS } \\
\text { DE LAS PUBLICACIONES }\end{array}$ \\
\hline \multirow[t]{5}{*}{$\begin{array}{c}\text { Enseñanza- } \\
\text { aprendizaje de } \\
\text { las Ciencias y de } \\
\text { la Biología }\end{array}$} & $\begin{array}{l}\text { Historia y epistemología de } \\
\text { la Biología }\end{array}$ & $\begin{array}{l}{ }^{*} \text { Historia de la enseñanza de la Biología en } \\
\text { Colombia. }\end{array}$ & $\begin{array}{l}\text { Chona, G.; Castaño, N.; Arteta, J; } \\
\text { (1998) Lo que nos dice la historia } \\
\text { de la enseñanza de la Biología en } \\
\text { Colombia. }\end{array}$ \\
\hline & $\begin{array}{l}\text { Educación para la salud - } \\
\text { Estudios en Calidad de vida }\end{array}$ & $\begin{array}{l}\text { *Los estudios de calidad de vida: Alternativa } \\
\text { de educación basada en la investigación. } \\
\text { *Mejoramiento de la calidad de vida en } \\
\text { relación con ruido en comunidades de } \\
\text { diferentes niveles educativos. }\end{array}$ & $\begin{array}{l}\text { Jessup, M. (1999) Evaluación de } \\
\text { Metodologías de Investigación } \\
\text { Interacción para el Desarrollo } \\
\text { Educativo. } \\
\text { Jessup, M.; Pulido, R. (1998) Los } \\
\text { estudios de calidad de vida. } \\
\text { Alternativa en Educación basada } \\
\text { en la investigación }\end{array}$ \\
\hline & $\begin{array}{l}\text { Educación en Ecología } \\
\text { colombiana }\end{array}$ & $\begin{array}{l}\text { Incorporación de nuevas tecnologías de la } \\
\text { información en el currículo del } \\
\text { Departamento de Biología Área Ecología. }\end{array}$ & $\begin{array}{l}\text { Rincón, M et al (2004) Trichoptera } \\
\text { del Santuario de Iguaque (Boyacá } \\
\text { Colombia) y su relación con la } \\
\text { calidad del agua. }\end{array}$ \\
\hline & $\begin{array}{l}\text { Educación para la } \\
\text { conservación de la } \\
\text { Biodiversidad }\end{array}$ & $\begin{array}{l}\text { Investigación en faunística y conservación, } \\
\text { con énfasis en los artrópodos. }\end{array}$ & $\begin{array}{l}\text { Torres, R. y García, M. (1998). Los } \\
\text { estudios faunísticos y de } \\
\text { bioproyección en la educación de } \\
\text { los colombianos. }\end{array}$ \\
\hline & Biotecnología y Educación & $\begin{array}{l}\text { *Incorporación de la Biotecnología al } \\
\text { currículo del Instituto Pedagógico Nacional. }\end{array}$ & $\begin{array}{l}\text { Valbuena, E.; (1998). Contribución } \\
\text { al desarrollo de biotecnología }\end{array}$ \\
\hline
\end{tabular}




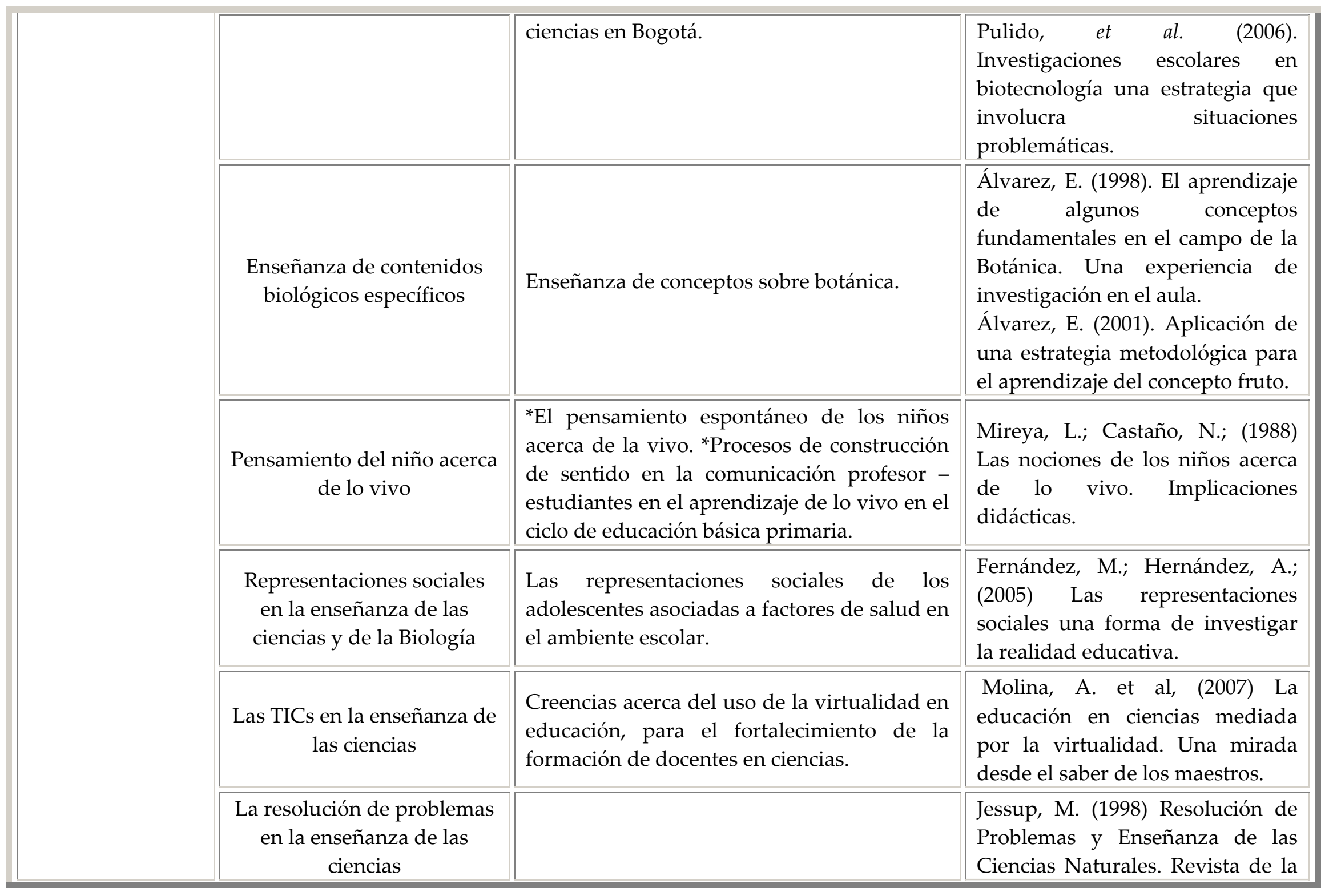




\begin{tabular}{|c|c|c|c|}
\hline & & & $\begin{array}{l}\text { Facultad de Ciencia y Tecnología } \\
\text { TEA: (3) 41-52. }\end{array}$ \\
\hline \multirow{3}{*}{$\begin{array}{l}\text { Estudios } \\
\text { biológicos }\end{array}$} & Ecología & $\begin{array}{l}\text { Trichoptera del Departamento de } \\
\text { Cundinamarca y su relación con la calidad } \\
\text { del agua. }\end{array}$ & Ruiz y Rincón (2002) \\
\hline & Biotecnología & $\begin{array}{l}\text { Implementación del cepario bacteriano del } \\
\text { DBI. }\end{array}$ & $\begin{array}{l}\text { Puentes, M.; et al (1999). } \\
\text { Aislamiento y caracterización de } \\
\text { cepas de Bacillus thuringiensis a } \\
\text { partir de suelo del municipio de } \\
\text { Mundo Nuevo (Cundinamarca). }\end{array}$ \\
\hline & Biodiversidad & $\begin{array}{l}\text { Investigación en faunística y conservación } \\
\text { con énfasis en los artrópodos. }\end{array}$ & $\begin{array}{l}\text { Torres, R. y Le Croom, (1997). } \\
\text { Una nueva especie de Pedaliodes } \\
\text { Butler, } 1867 \text { de la Cordillera } \\
\text { oriental de Colombia. Shilap }\end{array}$ \\
\hline \multirow{2}{*}{$\begin{array}{l}\text { Educación } \\
\text { ambiental }\end{array}$} & $\begin{array}{l}\text { La educación ambiental en } \\
\text { el contexto escolar }\end{array}$ & $\begin{array}{l}\text { Propuesta didáctica en educación ambiental } \\
\text { desde una perspectiva interdisciplinar para } \\
\text { incorporar la dimensión ambiental a la } \\
\text { escuela. }\end{array}$ & $\begin{array}{l}\text { Pérez, P.; Porras, Y.; (2007) } \\
\text { Identificación de las } \\
\text { representaciones de ambiente y } \\
\text { educación ambiental que circulan } \\
\text { en la escuela. }\end{array}$ \\
\hline & $\begin{array}{l}\text { La educación ambiental en } \\
\text { el contexto rural }\end{array}$ & $\begin{array}{l}\text { Aprendizajes significativos en ciencias } \\
\text { naturales, educación ambiental y educación } \\
\text { para la salud desde la perspectiva de aulas } \\
\text { alternativas en escuelas rurales }\end{array}$ & $\begin{array}{l}\text { Herrera, D. (1998) La granja } \\
\text { integral autosuficiente: estrategia } \\
\text { educativa de desarrollo sostenible } \\
\text { en el sector rural. }\end{array}$ \\
\hline \multirow{2}{*}{$\begin{array}{l}\text { Pensamiento y } \\
\text { Conocimiento } \\
\text { Profesional del } \\
\text { profesor de } \\
\text { Ciencias. }\end{array}$} & $\begin{array}{l}\text { Creencias de profesores de } \\
\text { Ciencias y de Biología }\end{array}$ & $\begin{array}{l}\text { Concepciones de ciencia, enseñanza y } \\
\text { aprendizaje en maestros de ciencia: } \\
\text { Incidencia en la educación científica en Santa } \\
\text { fe de Bogotá. }\end{array}$ & $\begin{array}{l}\text { Reyes L., Salcedo, L. E. y Perafán, } \\
\text { G. A. (2001) Creencias acerca del } \\
\text { cambio en la enseñanza de las } \\
\text { ciencias. }\end{array}$ \\
\hline & Pensamiento del profesor & Análisis del sistema de creencias que & Chona, G.; et al (2001) formas de \\
\hline
\end{tabular}




\begin{tabular}{|c|c|c|c|}
\hline & & & $\begin{array}{l}\text { competencias por parte de los } \\
\text { profesores de ciencias naturales. }\end{array}$ \\
\hline & $\begin{array}{l}\text { Conocimiento Profesional } \\
\text { del profesor de Biología }\end{array}$ & $\begin{array}{l}\text { Análisis de las concepciones de los docentes } \\
\text { en formación inicial acerca del Conocimiento } \\
\text { Profesional del profesor de Biología. }\end{array}$ & $\begin{array}{l}\text { Valbuena, Castro y Sierra (2007) } \\
\text { El Conocimiento Biológico desde } \\
\text { la perspectiva del Conocimiento } \\
\text { Profesional del Profesor de } \\
\text { Biología. }\end{array}$ \\
\hline \multirow{3}{*}{$\begin{array}{l}\text { Formación } \\
\text { docente }\end{array}$} & $\begin{array}{l}\text { Características de futuros } \\
\text { profesores de Biología e } \\
\text { implicaciones en la } \\
\text { formación docente. }\end{array}$ & $\begin{array}{l}\text { Condiciones de autonomía de los } \\
\text { estudiantes del Proyecto Curricular de } \\
\text { Licenciatura en Biología. }\end{array}$ & $\begin{array}{l}\text { Pérez, R. et al (2003) Las } \\
\text { condiciones de autonomía en los } \\
\text { estudiantes } \\
\text { Curricular de } \text { Licenciatura en } \\
\text { Biología. }\end{array}$ \\
\hline & $\begin{array}{l}\text { Autoevaluación curricular } \\
\text { en formación inicial }\end{array}$ & $\begin{array}{l}\text { Investigación evaluativo del Proyecto } \\
\text { Curricular de Licenciatura en Biología. }\end{array}$ & \\
\hline & $\begin{array}{l}\text { Competencias científicas en } \\
\text { docentes en ejercicio }\end{array}$ & $\begin{array}{l}\text { Desarrollo de competencias científicas y } \\
\text { formación en valores -Un estudio desde el } \\
\text { pensamiento del profesor de ciencias } \\
\text { experimentales- }\end{array}$ & $\begin{array}{l}\text { Arteta, J. et al (2005) La clase de } \\
\text { ciencias y la formación en valores. }\end{array}$ \\
\hline \multirow[t]{2}{*}{ Pedagogía } & Violencia y Escuela & $\begin{array}{l}\text { Violencia y escuela: efectos de la práctica } \\
\text { pedagógica en el mantenimiento, } \\
\text { trasformación y/o la generación de } \\
\text { manifestaciones o imaginarios de la } \\
\text { violencia en las instituciones escolares. }\end{array}$ & $\begin{array}{l}\text { Pinzón, et al. (2004) Axolotl. } \\
\text { Trayectos y aconteceres, un } \\
\text { proyecto sobre violencia y } \\
\text { escuela. }\end{array}$ \\
\hline & $\begin{array}{l}\text { El ser y el quehacer del } \\
\text { maestro }\end{array}$ & $\begin{array}{l}\text { Enseñanza de la Biología y la Pedagogía en } \\
\text { el DBI de la UPN }\end{array}$ & $\begin{array}{l}\text { Riveros (2006) Cómo se deja de } \\
\text { ser proyecto: Reflexión acerca de } \\
\text { los trayectos de la investigación. }\end{array}$ \\
\hline
\end{tabular}




\section{BIBLIOGRAFÍA}

- Arteta, J. Chona, G.; Arteta, J; Fonseca, G.; Ibáñez, X.; Martínez, S. (2005) La clase de ciencias y la formación en valores. Estudio de caso de cómo los profesores propician valores desde sus acciones. Revista Enseñanza de las ciencias. Número extra.

- Castaño, N., Chona, G., Pinzón, J., Lara, L., Valbuena, E., Martínez, S., Leudo, M., Arteta, J., Moncayo, G. y Herrera, D. 1998. Mirándonos para buscar sentido. TEA. Revista Facultad de Ciencia y Tecnología: UPN (4), 11-26.

- Chona, G.; Castaño, N.; Arteta, J; (1998) Lo que nos dice la historia de la enseñanza de la Biología en Colombia. Una aproximación. TEA. Revista de la Facultad de Ciencia y Tecnología: UPN (4) 5-10

- Chona, G.; Arteta, J; Fonseca, G.; Martínez, S.(2001) Formas de apropiación del discurso sobre competencias por parte de los profesores de ciencias naturales. TEA. Revista Facultad de Ciencia y Tecnología: UPN (9), 80-94.

- Fernández, M.; Hernández, A.; (2005) Las representaciones sociales una forma de investigar la realidad educativa. Revista Pedagogía y Saberes. Bogotá: UPN (23) 19-28

- Herrera, D. (1998) La granja integral autosuficiente: estrategia educativa de desarrollo sostenible en el sector rural. TEA. Revista Facultad de Ciencia y Tecnología: UPN (4) Revista digitalizada. .

- Jessup, M. (1999) Evaluación de Metodologías de Investigación - Interacción para el Desarrollo Educativo In: II Foro preparatorio al simposio nacional sobre investigación educativa Relaciones y Tensiones entre Innovación e Investigación Educativa, Enseñanza de las Ciencias y Tecnología, Bogotá. UPN y su aporte a las instituciones educativas. (1) 77-92

- Jessup, M.; Pulido, R. (1998) Los estudios de calidad de vida. Alternativa en Educación basada en la investigación. TEA. Revista de la Facultad de Ciencia y Tecnología: UPN (4) 111124.

- Jessup, M. (1998) Resolución de Problemas y Enseñanza de las Ciencias Naturales. TEA. Revista de la Facultad de Ciencia y Tecnología: UPN (3) 41-52

- Martínez, S, Bernal, A, Gómez, A, Ortiz, E, Zafra, S, Ibáñez, S, Pedraza, M, Oicatá, M, Foseca, G, Arteta, J. (2003). De la pregunta al proyecto. En: Emociones y Razones para innovar en la Enseñanza de las Ciencias. Siete experiencias pedagógicas de la Escuela Básica. Alcaldía Mayor de Bogotá. Instituto para la Investigación Educativa y Desarrollo Pedagógico (IDEP).

- Pinzón, J, Rodríguez, L, Riveros, o, Roa, p., Zipaquirçá, L.(2004). Axolotl. Trayectos y aconteceres, un proyecto sobre violencia y escuela. Bogotá: Universidad Pedagógica Nacional.

- Reyes L., Salcedo, L. E. y Perafán, G. A (2001) Creencias acerca del cambio en la enseñanza de las ciencias. En: Enseñanza de las ciencias, número extra, VI congreso Tomo I.: 369.

- Rincón, M.; Medellín, F.; Ramírez, M. (2004) Trichoptera del Santuario de Iguaque (Boyacá Colombia) y su relación con la calidad del agua. Revista Colombiana de Entomología: Guadalupe Ltda. (30)2,197-203

- Riveros, O. (2006). Cómo se deja de ser proyecto: Reflexión acerca de los trayectos de la investigación. En: Los bordes de la pedagogía. Universidad Pedagógica Nacional Instituto Nacional de Pedagogía. 35-61 
- Ruiz, V. y Rincón, M. (2002). Calidad ecológica del agua del río Villeta. TEA. Revista Facultad de Ciencia y Tecnología. No. 12

- Torres, R. y García, M. (1998). Los estudios faunísticos y de bioproyección en la educación de los colombianos. TEA. Revista Facultad de Ciencia y Tecnología. No. (14) Revista digitalizada.

- Torres, R. y Le Croom, (1997). Una nueva especie de Pedaliodes Butler, 1867 de la Cordillera oriental de Colombia. Shilap Revista de Lepidopterología Vol 25 (100): 213-218.

- Mireya, L.; Castaño, N.; (1988) Las nociones de los niños acerca de lo vivo. Implicaciones didácticas. TEA. Revista de la Facultad de Ciencia y Tecnología: UPN (4) 49 - 57

- Molina, A.; Ortiz, G.; Waltero, C.; (2007) La educación en ciencias mediada por la virtualidad. Una mirada desde el saber de los maestros. En: Investigación e innovación en la enseñanza de las ciencias. Bogotá: Universidad Católica de Colombia. Revista digital.

- Pérez, P.; Porras, Y.; (2007) Identificación de las representaciones de ambiente y educación ambiental que circulan en la escuela. TEA. Revista de la Facultad de Ciencia y Tecnología: UPN (21) Revista digitalizada

- Pérez, R. Mireya, L.; Martínez, S.; Velasco, A.; Martínez, S. Pedraza, M.; (2003) Las condiciones de autonomía en los estudiantes del Proyecto Curricular de Licenciatura en Biología. TEA. Revista Facultad de Ciencia y Tecnología. Bogotá: V.Extra, 124 - 126.

- Programa de Licenciatura en Biología. Nuevo plan de estudios. Junio 1984.

Departamento de Biología Universidad Pedagógica Nacional. Documento de circulación interna.

- Puentes, M.; Mozo, N.; Valbuena, E. (1999). Aislamiento y caracterización de cepas de Bacillus thuringiensis a partir de suelo del municipio de Mundo Nuevo (Cundinamarca). Revista de la Asociación Colombiana de Ciencias Biológicas ACCB Vol. 11 No. 1, pag 94 - 102.

- Puerto, L.; Rodríguez, L. (1995). Resumen de los trabajos de grado y tendencias investigativas (1989 - 1994), Departamento de Biología, Universidad Pedagógica Nacional. Trabajo de grado

- Pulido, M.; Acosta, R.; García, Y.; Bolaño, P.; Valbuena, E.; López, S.; Chavarro, C. (2006) Investigaciones escolares en biotecnología una estrategia que involucra situaciones problemáticas. En: Ciencias, racionalidades y medio Ambiente. Bogotá: Editorial Pontificia Universidad Javeriana.

- Valbuena, E. (1998). Contribución al desarrollo de biotecnología desde la educación en los niveles de básica y media. TEA. Revista Facultad de Ciencia y Tecnología: UPN (4) Revista digitalizada.

- Valbuena, E., Castro A., y Sierra, C. (2007) El Conocimiento Biológico desde la perspectiva del Conocimiento Profesional del Profesor de Biología. Revista de la Asociación de Docentes de Ciencias Biológicas de Argentina (en prensa). 